\title{
Aarioo $\mid$ Fatores associados à notificação de perda auditiva induzida por ruído no Brasil, 2013-2015: estudo ecológico*
}

doi: 10.1590/S1679-49742021000200018

\author{
Factors associated with notification of noise-induced hearing loss in Brazil, 2013-2015: an \\ ecological study
}

Factores asociados a la notificación de pérdida auditiva inducida por ruido en Brasil, 2013-2015: estudio ecológico

\author{
Aline Cristina Gusmão' - (1) orcid.org/0000-0001-7624-4114 \\ Tatiane Costa Meira' ${ }^{1}$ - (D) orcid.org/0000-0002-9730-1621 \\ Silvia Ferrite ${ }^{2}$ - (1) orcid.org/0000-0001-5344-3751 \\ 'Universidade Federal da Bahia, Instituto de Saúde Coletiva, Salvador, BA, Brasil \\ ${ }^{2}$ Universidade Federal da Bahia, Instituto de Ciências da Saúde, Salvador, BA, Brasil
}

\section{Resumo}

Objetivo: Investigar fatores associados à notificação de perda auditiva induzida por ruído (Pair), no Sistema de Informação de Agravos de Notificação no Brasil. Métodos: Estudo ecológico para estimar a proporção de municípios notificantes de Pair. Foram empregados modelos de regressão logística para identificar fatores associados. Resultados: Entre 2013 e 2015, 277 $(5,0 \%)$ municípios notificaram Pair. A notificação foi mais comum entre municípios com cobertura por Cerest $(0 \mathrm{R}=1,62-$ $\left.\mathrm{IC}_{95 \%} 1,02 ; 2,59\right)$ ou que sediavam unidade de Cerest em seu território $\left(0 \mathrm{R}=4,37\right.$ - $\left.\mathrm{IC}_{95 \%} 2,75 ; 6,93\right)$, a menor distância da capital do estado $\left(0 \mathrm{R}=1,43-\mathrm{IC}_{95 \%} 1,06 ; 1,92\right)$ e com alto índice de desenvolvimento humano $\left(0 \mathrm{R}=2,35-\mathrm{IC}_{95 \%} 1,16 ; 4,75\right)$. Entre os municípios situados em área com cobertura de Cerest, a notificação foi mais comum quando na equipe havia fonoaudiólogo $\left(0 \mathrm{R}=1,96-\mathrm{IC}_{95 \%} 1,47 ; 2,63\right)$ e era baixa a rotatividade de profissionais $\left(0 \mathrm{R}=1,88-\mathrm{IC}_{95 \%} 1,40 ; 2,52\right)$. Conclusão: Fatores contextuais influenciam na notificação de Pair, notadamente a existência e qualificação dos Cerest.

Palavras-chave: Perda Auditiva Induzida por Ruído; Sistemas de Informação em Saúde; Vigilância em Saúde Pública; Saúde do Trabalhador; Estudos Ecológicos.

\footnotetext{
*Artigo derivado de dissertação de mestrado intitulada 'Fatores contextuais associados à notificação de perda auditiva induzida por ruído no Sinan no Brasil', defendida por Aline Cristina Gusmão Souza Gomes junto ao Programa de Pós-Graduação em Saúde Coletiva da Universidade Federal da Bahia em 24 de julho de 2019. O estudo contou com o apoio financeiro da Coordenação de Aperfeiçoamento de Pessoal de Nível Superior/Ministério da Educação (Capes/MEC), processo nº 001.
}

Endereço para correspondência:

Aline Cristina Gusmão - Universidade Federal da Bahia, Instituto de Saúde Coletiva, Programa Integrado em Saúde Ambiental e do Trabalhador, Rua Basílio da Gama, S/N, Canela, Salvador, BA, Brasil. CEP: 40110-040

E-mail: aline.cristina@ufba.br 


\section{Introdução}

A perda auditiva induzida por ruído (Pair) é um dos problemas de saúde ocupacional mais disseminados e prevalentes no mundo. ${ }^{1-4}$ De acordo com a Organização Mundial da Saúde (OMS), no ano 2000, a Pair era responsável por 19,0\% dos anos vividos com incapacidade causada por todas as doenças e agravos decorrentes de fatores ocupacionais, em todo o mundo. ${ }^{5}$ Listada na Classificação Estatística Internacional de Doenças e Problemas Relacionados à Saúde - $10^{2}$ Revisão (CID-10), a Pair é referida pelo código H83.3.

\section{A perda auditiva induzida por ruído (Pair) é um dos problemas de saúde ocupacional mais disseminados e prevalentes no mundo. De acordo com a Organização Mundial da Saúde (OMS), no ano 2000, a Pair era responsável por $19,0 \%$ dos anos vividos com incapacidade causada por todas as doenças e agravos decorrentes de fatores ocupacionais.}

No Brasil, a Pair é um agravo de notificação compulsória no Sistema de Informação de Agravos de Notificação (Sinan), a ser monitorado pela Vigilância em Saúde do Trabalhador (VISAT). ${ }^{6} 0$ Sistema Único de Saúde (SUS), responsável pela VISAT, criou em 2002 a Rede Nacional de Atenção Integral à Saúde do Trabalhador (Renast), da qual fazem parte 210 Centros de Referência em Saúde do Trabalhador (Cerest) responsáveis pela coordenação das ações em regiões, cada uma abrangendo um conjunto de municípios. ${ }^{6}$ Uma das atribuições do Cerest é agir como um centro especializado de apoio à prevenção, promoção e cuidados de saúde, garantindo a notificação de agravos à saúde relacionados ao trabalho nos sistemas de informaçoes administrados pelo Departamento de Informática do SUS (Datasus), entre eles o Sinan. ${ }^{7}$ Apesar do tempo transcorrido desde sua inclusão em 2004, 0 número de casos de Pair notificados no Sinan ainda é pequeno ${ }^{8}$ considerando-se sua prevalência encontrada em estudos conduzidos com amostras ou populações de empresas específicas. ${ }^{910}$
Não foram encontrados estudos sobre fatores associados à notificação de Pair e sim sobre os fatores que aumentaram a notificação de acidentes de trabalho no Sinan (2008-2009), no âmbito do Cerest: infraestrutura adequada; maior quantidade e oferta de capacitação de pessoal; e atendimento a demandas externas. ${ }^{11}$ No município de Fortaleza, capital do estado do Ceará, também foram identificados como características favoráveis à notificação de acidentes de trabalho, entre 2013 e 2014, o tempo de atuação dos profissionais nas unidades sentinela, a participação em treinamentos e capacitações relacionados aos acidentes de trabalho, o conhecimento sobre portarias e a Legislação que dão suporte à notificação, a compreensão do caráter compulsório das notificações, e discussões sobre o tema no cotidiano laboral. ${ }^{12}$

0 conhecimento sobre esses fatores pode subsidiar ações que favoreçam a notificação da Pair no Sinan, colaborando para sua viabilidade como estratégia de vigilância do agravo no país. Este estudo teve como objetivo investigar fatores contextuais associados à notificação de Pair no Brasil.

\section{Métodos}

Trata-se de estudo ecológico sobre fatores associados à notificação de Pair no Brasil, no período entre 2013 e 2015. Segundo dados do Instituto Brasileiro de Geografia e Estatística (IBGE), o país contava com 202.768.562 habitantes em 2014, e sua população economicamente ativa e ocupada - doravante chamada de população ocupada - correspondia a 99,4 milhões de trabalhadores $(49,0 \%) .{ }^{13}$ Foram unidades de observação todos os 5.570 municípios existentes no período, distribuídos entre as 27 Unidades da Federação (UFs).

Uma vez identificados todos os casos de Pair notificados no período estudado, por município, definiu-se a variável de desfecho: município notificante-'sim' (1)-, quando apresentava pelo menos uma notificação de Pair; e município 'não' notificante - (0) -, na ausência de notificação. A variável 'fonte notificadora' foi utilizada para indicar se as notificações do município foram registradas exclusivamente via Cerest, apenas por outras vias (exceto Cerest), ou por ambas as fontes.

As variáveis analisadas como potencialmente preditoras para o desfecho foram:

a) Cobertura por Cerest (sim; não);

b) Município-sede de Cerest (sim; não); 
c) Macrorregião do país (Norte; Nordeste; Sudeste; Sul; Centro-Oeste);

d) UF;

e) Distância do município à capital da UF em quilômetros $(\mathrm{km})$, calculada por uma reta a partir do centroide das coordenadas geográficas do município e ajustada com o fator euclidiano, categorizada pela mediana em $(0) \geq 275$ ou (1) $<275$;

f) Extensão territorial em quilômetros quadrados $\left(\mathrm{km}^{2}\right)$, categorizada com base na mediana em (0) $\leq 418$ e $(1)>418$;

g) População ocupada, com base no Censo Demográfico de 2010 ( $\leq 5 \mathrm{mil} ;>5$ a $20 \mathrm{mil} ;>20$ a 50 mil; > $>0$ a 100 mil; >100 mil);

h) Trabalhadores nas indústrias de transformação/ extrativa/construção, que representa a proporção dos trabalhadores no município nesses ramos de atividade, categorizados em $<15,0 \%$ ou $\geq 15,0 \%$;

i) Trabalhadores na indústria de transformação, categorizados pela proporção $\leq 6,4 \%$ ou $>6,4 \%$, com base na mediana;

j) Trabalhadores na indústria extrativa, categorizados em $\leq 0,1 \%$ ou $>0,1 \%$, com base na mediana;

k) Trabalhadores na construção, categorizados em $\leq 6,0 \%$ ou $>6,0 \%$, com base na mediana;

1) Trabalhadores com emprego formal, categorizados com base na mediana, em $\leq 34,1 \%$ ou $>34,1 \%$, considerando-se como emprego formal os trabalhadores com carteira assinada, militares, funcionários públicos estatutários e empregadores, e como não formais os trabalhadores por conta própria, na produção para o próprio consumo, não remunerados e empregados sem carteira assinada;

m) Índice de desenvolvimento humano municipal (IDH-M), tendo como referência 0 ano de 2010, categorizado em baixo/muito baixo $(0,000$ a $0,599)$, médio $(0,600$ a 0,699$)$ ou alto/muito alto $(0,700$ a 1,000$)$, com base na classificação proposta pelo Atlas do Desenvolvimento Humano no Brasil. $^{14}$

Para os municípios com cobertura, ou seja, pertencentes à área de abrangência de um determinado Cerest, foram também analisadas as seguintes variáveis:

a) Ano de habilitação do Cerest, categorizado pela mediana, $\leq 2005$ ou >2005; b) Número médio de vínculos da equipe do Cerest, calculado pela média dos vínculos CNES referentes a 2013 e 2015 , categorizado como $<10$ ou $\geq 10$, com base na legislação vigente à época, que estabelecia os recursos humanos mínimos para os Cerest regionais;

c) Proporção da equipe com vínculo estatutário, categorizada em $\leq 50 \%$ ou $>50 \%$;

d) Número médio de fonoaudiólogos na equipe do Cerest, calculado pela média do número de profissionais fonoaudiólogos no CNES em 2013 e 2015, categorizado como $<1$ ou $\geq 1$;

f) Rotatividade na composição da equipe no transcorrer do período 2013-2015, definida a partir de dados nominais do CNES, considerando-se 'maior rotatividade' quando mais de um terço dos profissionais da equipe do Cerest em 2013 não constavam da relação de profissionais da equipe em 2015, e 'menor rotatividade' quando isso ocorria para no máximo um terço dos profissionais.

Constituíram fontes de dados do estudo:

a) IBGE, ${ }^{15}$ sobre 0 ano de 2018, para obter a lista e códigos dos municípios brasileiros e sua extensão territorial; e sobre 2010, para obter o tamanho da população ocupada, a proporção dos trabalhadores com emprego formal, o tamanho e proporção dos trabalhadores na indústria extrativa/transformação/construção e o IDH-M;

b) Google Maps, ${ }^{16}$ sobre 0 ano de 2018, para obter as coordenadas geográficas dos municípios;

c) Banco de notificações de Pair no Sinan (atualização em agosto de 2018), cedido pela Coordenação-Geral de Saúde do Trabalhador do Ministério da Saúde (CGSAT/MS) ao Centro Colaborador de Vigilância aos Agravos à Saúde do Trabalhador do Programa Integrado em Saúde Ambiental e do Trabalhador, Instituto de Saúde Coletiva, Universidade Federal da Bahia ${ }^{17}$ para obter a lista de casos notificados de Pair, o ano-calendário da notificação, a fonte notificadora e o município de residência;

d) Documento sobre o histórico dos Cerest, cedido pela CGSAT/MS em janeiro de 2018, para acessar 0 ano calendário de habilitação e desabilitação de cada Cerest;

e) Website Renast Online ${ }^{18}$ (2014) e documento complementar (outubro de 2017), cedido pela 
CGSAT/MS, para identificar os municípios na área de abrangência de cada Cerest;

f) Cadastro Nacional de Estabelecimentos de Saúde $(\mathrm{CNES})^{19}$ referente ao período 2013-2015, para obter a composição das equipes dos Cerest.

Para verificar se as variáveis estavam associadas à ocorrência de notificação de Pair nos municípios, foram descritos (i) a quantidade e a proporção de municípios notificantes por ano-calendário e para todo 0 período 2013-2015, de acordo com as variáveis preditoras; foram estimadas (ii) as medidas de associação entre cada uma das variáveis com o desfecho (município notificante), empregando-se a regressão logística para obtenção da razão de chances (odds ratio, OR) bruta e ajustada pelo tamanho da população ocupada (ORaj), e respectivos intervalos de confiança de $95 \%$ $\left(\mathrm{IC}_{95 \%}\right)$; e foi conduzida (iii) a análise multivariada, separadamente para os conjuntos de variáveis relacionadas aos municípios e relacionadas aos Cerest. $\mathrm{Na}$ modelagem, além da população ocupada, permaneceram as variáveis que apresentaram p-valor $<0,20$, tendo seguido para os respectivos modelos finais de cada dimensão aquelas variáveis associadas ao desfecho considerando-se um alfa $\leq 0,05$.

A base de dados foi organizada a partir de planilhas Excel® e as análises foram conduzidas utilizando-se o programa estatístico Stata, versão 12.0. 0 programa QGIS versão 2.6, de georreferenciamento, foi utilizado

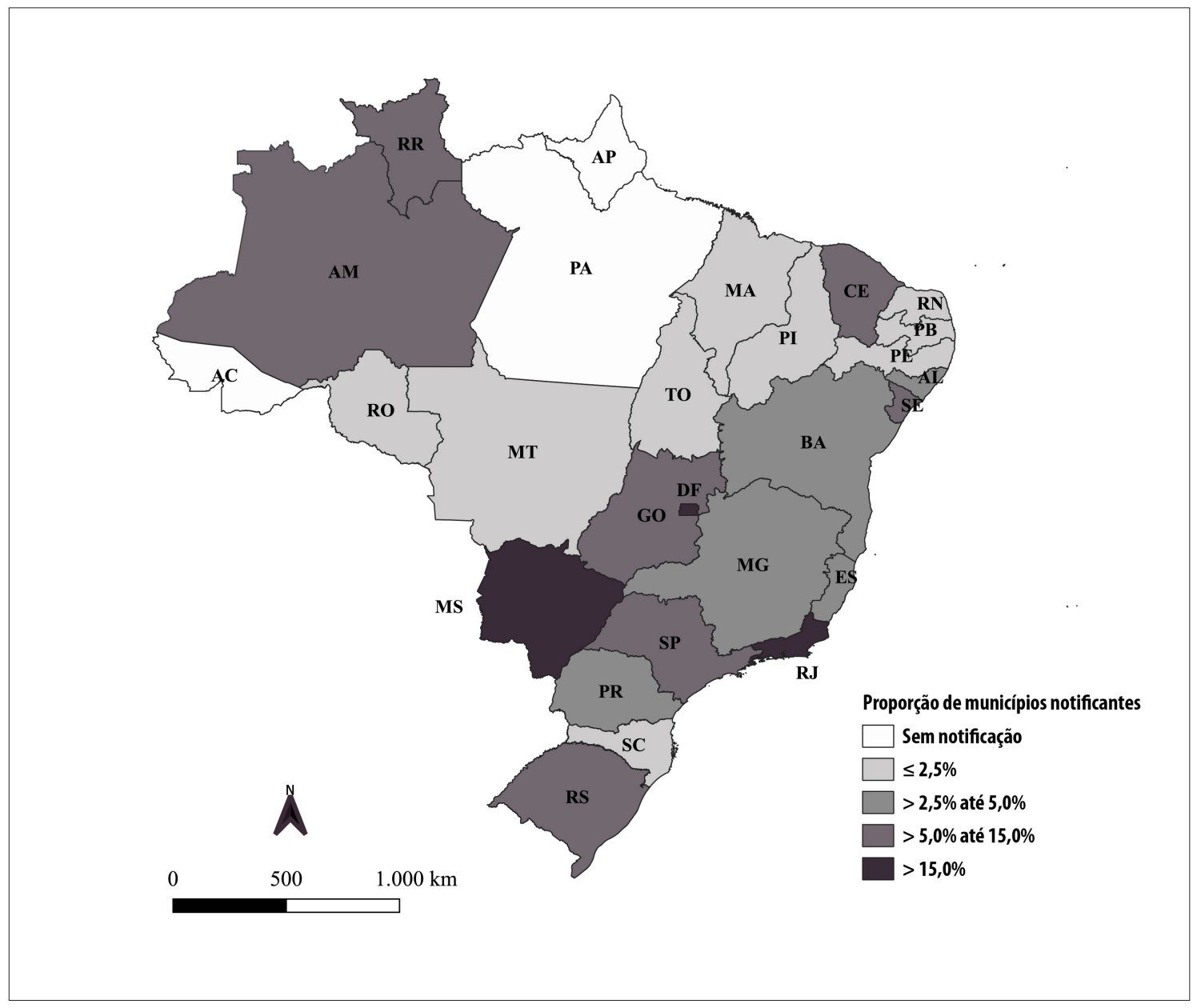

Legenda: AC: Acre; AL: Alagoas; AM: Amazonas; AP: Amapá; BA: Bahia; CE: Ceará; DF: Distrito Federal; ES: Espírito Santo; GO: Goiás; MA: Maranhão; MG: Minas Gerais; MS: Mato Grosso do Sul; MT: Mato Grosso; PA: Pará; PB: Paraíba; PE: Pernambuco; PI: Piauí; PR: Paraná; RJ: Rio de Janeiro; RN: Rio Grande do Norte; RO: Rondônia; RR: Roraima; RS: Rio Grande do Sul; SC: Santa Catarina; SE: Sergipe; SP: São Paulo; TO: Tocantins.

\section{Figura 1 - Proporção de municípios ( $\mathrm{N}=5.570$ ) que notificaram pelo menos um caso de perda auditiva induzida por ruído no Sistema de Informação de Agravos de Notificação, segundo Unidades da Federação, Brasil, 2013-2015}


para a construção do mapa. 0 projeto do estudo foi aprovado pelo Comitê de Ética em Pesquisa do Instituto de Saúde Coletiva da Universidade Federal da Bahia (CEP/ ISC/UFBA): Certificado de Apresentação para Apreciação Ética (CAAE) n n 93708218.0.0000.5030, Parecer $\mathrm{n}^{0}$ 2.799.319, 03/08/2018. Não foi necessário aplicar Termo de Consentimento Livre e Esclarecido, pela natureza pública, agregada e/ou anônima dos dados.

\section{Resultados}

Dos 5.570 municípios brasileiros, 277 (5,0\%) notificaram casos de Pair no Sinan no período, evoluindo de 113 (2,0\%), em 2013, para 164 municípios (2,9\%) em 2015: um crescimento de 45,1\%. Entre as UFs, Mato Grosso do Sul apresentou a maior proporção de municípios notificantes (25,3\%), seguida do Rio de Janeiro (18,5\%) e São Paulo (10,1\%), enquanto no Acre, Amapá e Pará nenhum município notificou Pair no período (Figura 1). Entre os municípios notificantes, $42,0 \%$ fizeram-no unicamente via Cerest, 52,8\% unicamente por outras fontes de notificação, e 5,2\% notificaram tanto via Cerest como por outras fontes.

Considerando-se a totalidade dos municípios, 4.400 (79,0\%) pertenciam a áreas de abrangência de Cerest, sendo 3,2\% municípios-sede; a maioria dos municípios tinha até 5 mil trabalhadores enquanto população ocupada; e menos de 15,0\% dos trabalhadores encontravam-se na indústria de transformação, extrativa ou da construção. Um quarto dos municípios classificava-se na faixa de IDH-M muito baixo/baixo, entre outras características (Tabela 1). Dos 4.400 municípios localizados na área de abrangência de um Cerest, a maioria contava com essa unidade habilitada até 0 ano de 2005, número médio de vínculos na equipe de pelo menos dez profissionais, mais da metade da equipe composta por estatutários e número médio de fonoaudiólogos menor que um profissional, no período; e com rotatividade da equipe maior que 33,3\% (Tabela 2).

A proporção de municípios notificantes de Pair no Sinan elevou-se com o aumento do tamanho da população ocupada, passando de $1,4 \%$, no grupo de municípios com população ocupada $\leq 5$ mil hab., para $4,0 \%, 11,0 \%, 33,3 \%$ e $56,0 \%$, respectivamente, para população ocupada $>5$ até 20 mil, $>20$ a 50 mil, $>50$ a 100 mil e $>100$ mil hab. Esta variável foi utilizada para ajuste das $\mathrm{OR}$ em todas as análises.
$\mathrm{Na}$ análise bivariada, todas as variáveis relacionadas aos municípios foram associadas ao desfecho (Tabela 3). Na modelagem para essa dimensão, foram conduzidos dois modelos, separadamente: um incluindo unicamente a variável 'trabalhadores nas indústrias de transformação/extrativa/construção', e outro com cada uma das três variáveis de trabalhadores dessas indústrias, visto que se tratava de dados de mesma origem, induzindo colinearidade. Em ambos os modelos, uma maior proporção da população ocupada nesses ramos de atividade não se revelou fator associado à notificação da Pair pelos municípios. No modelo final, mantiveram-se associados positivamente ao desfecho: ter cobertura por Cerest; ser sede de Cerest; pertencer à região Centro-0este; apresentar menor distância da capital; e ter IDH-M alto/muito alto (Tabela 3). Pertencer à região Norte foi negativamente associado ao desfecho.

Todas as variáveis relacionadas aos Cerest foram associadas ao desfecho na análise bivariada, exceto a proporção de componentes da equipe com vínculo estatutário (Tabela 4). No modelo final, mantiveram-se associados positivamente ao desfecho: ter pelo menos um fonoaudiólogo na equipe do Cerest; e esta apresentar menor rotatividade de profissionais (Tabela 4).

\section{Discussão}

No Brasil, entre 2013 e 2015, poucos municípios notificaram Pair no Sinan. 0s fatores que se associaram positivamente à condição de município notificante foram ser município-sede ou ter cobertura por Cerest, estar próximo à capital do estado e ter alto IDH-M. Enquanto municípios localizados na região Centro-0este foram mais comumente notificantes de Pair, na comparação com outras regiões, o contrário foi observado na região Norte. Entre os municípios com cobertura, ou seja, situados em área de abrangência de um Cerest, os fatores que influíram positivamente na notificação de Pair foram a baixa rotatividade de profissionais e a presença de fonoaudiólogo na equipe do Cerest.

Embora apenas um em cada 20 municípios tenha notificado Pair no período do estudo, o crescimento dos municípios notificantes entre 2013 e 2015 foi expressivo. Tal crescimento é compatível com a implantação e expansão dos Cerest no Brasil. Houve aumento no número de Cerest desde 2002, quando foram habilitados os primeiros 17 no país. ${ }^{6,20}$ Contudo, mesmo 
Tabela 1 - Distribuição dos municípios ( $\mathrm{N}=5.570$ ) segundo variáveis contextuais relacionadas ao município, Brasil, 2013-2015

\begin{tabular}{|c|c|c|}
\hline Variáveis & N & $\%$ \\
\hline \multicolumn{3}{|c|}{ Cobertura por Cerest ${ }^{\mathrm{a}}$} \\
\hline Não & 1.170 & 21,0 \\
\hline Sim & 4.400 & 79,0 \\
\hline \multicolumn{3}{|l|}{ Sede de Cerest ${ }^{\mathrm{a}}$} \\
\hline Não & 5.392 & 96,8 \\
\hline Sim & 178 & 3,2 \\
\hline \multicolumn{3}{|l|}{ Macrorregião } \\
\hline Norte & 450 & 8,1 \\
\hline Nordeste & 1.794 & 32,2 \\
\hline Sudeste & 1.668 & 29,9 \\
\hline Sul & 1.191 & 21,4 \\
\hline Centro-0este & 467 & 8,4 \\
\hline \multicolumn{3}{|c|}{ Distância até a capital (km) } \\
\hline$\geq 275,0$ & 2.781 & 49,9 \\
\hline$<275,0$ & 2.789 & 50,1 \\
\hline \multicolumn{3}{|c|}{ Extensão territorial $\left(\mathbf{k m}^{2}\right)$} \\
\hline$\leq 418$ & 2.785 & 50,0 \\
\hline$>418$ & 2.785 & 50,0 \\
\hline \multicolumn{3}{|c|}{ População ocupada (trabalhadores) ${ }^{b}$} \\
\hline$\leq 5.000$ & 2.942 & 52,9 \\
\hline 5.001 a 20.000 & 1.973 & 35,4 \\
\hline 20.001 a 50.000 & 399 & 7,2 \\
\hline 50.001 a 100.000 & 126 & 2,3 \\
\hline$>100.000$ & 125 & 2,2 \\
\hline \multicolumn{3}{|c|}{ Trabalhadores nas indústrias de transformação/extrativa/construção (\%) } \\
\hline$<15,0$ & 2.974 & 53,4 \\
\hline$\geq 15,0$ & 2.596 & 46,6 \\
\hline \multicolumn{3}{|c|}{ Trabalhadores na indústria de transformação (\%) } \\
\hline$\leq 6,4$ & 2.785 & 50,0 \\
\hline$>6,4$ & 2.785 & 50,0 \\
\hline \multicolumn{3}{|c|}{ Trabalhadores na indústria extrativa (\%) } \\
\hline$\leq 0,1$ & 2.871 & 51,5 \\
\hline$>0,1$ & 2.699 & 48,5 \\
\hline \multicolumn{3}{|c|}{ Trabalhadores na construção (\%) } \\
\hline$\leq 6,0$ & 2.796 & 50,2 \\
\hline$>6,0$ & 2.774 & 49,8 \\
\hline \multicolumn{3}{|c|}{ Trabalhadores com emprego formal (\%) } \\
\hline$\leq 34,1$ & 2.780 & 50,0 \\
\hline$>34,1$ & 2.785 & 50,0 \\
\hline
\end{tabular}


Continuação

Tabela 1 - Distribuição dos municípios ( $\mathrm{N}=5.570)$ segundo variáveis contextuais relacionadas ao município, Brasil, $2013-2015$

\begin{tabular}{llc}
\hline Variáveis & N & $\%$ \\
\hline Índice de desenvolvimento humano municipal (IDH-M) & & \\
\hline Baixo/muito baixo & 1.399 & 25,1 \\
Médio & 2.263 & 40,7 \\
Alto/muito alto & 1.903 & 34,2 \\
\hline
\end{tabular}

a) Cerest: (entro de Referência em Saúde do Trabalhador; b) Cinco dados faltantes porque os municípios foram criados após o Censo Demográfico de 2010.

Tabela 2 - Distribuição dos municípios com cobertura por Cerest ${ }^{\mathrm{a}}(\mathrm{N}=4.400)$ segundo variáveis contextuais relacionadas ao Cerest $^{\text {a }}$ e sua equipe, Brasil, 2013-2015

\begin{tabular}{lcc}
\hline Variáveis & N & $\%$ \\
\hline Ano de habilitação do Cerest ${ }^{\text {a }}$ e referência & & \\
\hline$>2005$ & 2.158 & 49,1 \\
$\leq 2005$ & 2.242 & 50,9 \\
\hline Número médio de vínculos (2013-2015) & 1.557 & 35,4 \\
\hline$<10$ & 2.843 & 64,6 \\
$\geq 10$ & & 43,8 \\
\hline Componentes da equipe com vínculo estatutário (\%) & 1.926 & 56,2 \\
\hline$\leq 50,0$ & 2.474 & 64,5 \\
$>50,0$ & & 35,5 \\
\hline Número médio de fonoaudiólogos (2013-2015) & 2.837 & 1.563 \\
\hline$<1$ & & 52,8 \\
\hline 1 & & 2.261 \\
\hline Motatividade na equipe
\end{tabular}

a) Cerest: Centro de Referência em Saúde do Trabalhador; b) 119 dados faltantes pela ausência dos registros para 2013 e/ou 2015, ambos necessários para 0 cálculo.

alcançando 210 unidades em $2011,{ }^{21} 1.170$ municípios brasileiros ainda não estavam cobertos por Cerest no período deste estudo.

Em três estados, Acre, Amapá e Pará, não houve municípios notificantes de Pair entre 2013 e 2015, em contraste com Mato Grosso do Sul, Rio de Janeiro e São Paulo, UFs com as maiores proporções de municípios notificantes. Nota-se que, no período 20072009 , as notificações de Pair eram ausentes $(n=8)$ ou inferiores a dez casos notificados $(n=12)$ em 20 das 27 UF. $^{8}$ Pode-se inferir que houve uma evolução, embora existissem situações bastante distintas no território nacional. Na região Sudeste, de importante atividade industrial, observa-se o desenvolvimento de programas e ações sistemáticas em saúde do trabalhador, ${ }^{20}$ expressiva produção científica na área ${ }^{22}$ e um histórico mais precoce de habilitação dos Cerest. ${ }^{23}$ São Paulo foi o primeiro estado a apresentar uma rede de Cerest regionais habilitados, ${ }^{23} \mathrm{e}$ dos 17 primeiros Cerest implantados em 2002, seis (35,3\%) localizavam-se no estado paulista e os demais em Minas Gerais, Rio Grande do Sul e Bahia. ${ }^{6}$ Nas regiões com maior densidade de trabalhadores - Sudeste, Sul e Nordeste -, observa-se maior concentração de Cerest, ao passo que, nas regiões Norte e Centro-Oeste, há uma rarefação de unidades. ${ }^{20}$ Apesar disso, o Centro-0este destacou-se com a maior proporção de municípios notificantes de Pair, possível consequência de uma implantação bem 
Tabela 3 - Proporção de municípios ( $\mathrm{N}=5.570)$ que notificaram perda auditiva induzida por ruído (Pair) no Sistema de Informação de Agravos de Notificação, razão de chances e análise multivariada, conforme variáveis contextuais relacionadas ao município, Brasil, 2013-2015

\begin{tabular}{|c|c|c|c|c|c|c|c|c|c|}
\hline \multirow{3}{*}{ Variáveis } & \multirow{3}{*}{$\mathbf{N}$} & \multirow{2}{*}{\multicolumn{2}{|c|}{ Notificaram Pair }} & \multicolumn{4}{|c|}{ Medida de associação } & \multirow{2}{*}{\multicolumn{2}{|c|}{ Modelo finala }} \\
\hline & & & & \multicolumn{2}{|c|}{ Bruta } & \multicolumn{2}{|c|}{$\begin{array}{c}\text { Ajustada pela } \\
\text { população ocupada }\end{array}$} & & \\
\hline & & N & $\%$ & $\mathbf{O} \mathbf{R}^{\mathrm{b}}$ & $\mathrm{IC}_{95 \%} \mathrm{C}^{\mathrm{C}}$ & $\mathbf{O} \mathbf{R}^{\mathrm{b}}$ & IC $_{95 \%} \mathrm{C}^{\mathrm{C}}$ & $\mathrm{OR}^{\mathrm{b}}$ & IC $_{95 \%} \mathrm{C}^{\mathrm{C}}$ \\
\hline Brasil & 5.570 & 277 & 5,0 & & & & & & \\
\hline \multicolumn{10}{|c|}{ Cobertura por Cerest $^{d}$} \\
\hline Não & 1.170 & 23 & 2,0 & 1,00 & & 1,00 & & 1,00 & \\
\hline Sim & 4.400 & 254 & 5,8 & 3,06 & 1,$98 ; 4,70$ & 1,81 & 1,$15 ; 2,83$ & 1,62 & 1,$02 ; 2,59$ \\
\hline \multicolumn{10}{|c|}{ Sede de Cerest ${ }^{d}$} \\
\hline Não & 5.392 & 186 & 3,4 & 1,00 & & 1,00 & & 1,00 & \\
\hline Sim & 178 & 91 & 51,1 & 29,28 & 21,$08 ; 40,65$ & 3,90 & 2,$52 ; 6,05$ & 4,37 & 2,$75 ; 6,93$ \\
\hline \multicolumn{10}{|c|}{ Macrorregião } \\
\hline Norte & 450 & 7 & 1,6 & 0,47 & 0,$21 ; 1,04$ & 0,35 & 0,$15 ; 0,81$ & 0,32 & 0,$13 ; 0,79$ \\
\hline Nordeste & 1.794 & 58 & 3,2 & 1,00 & & 1,00 & & 1,00 & \\
\hline Sudeste & 1.668 & 124 & 7,4 & 2,40 & 1,$75 ; 3,31$ & 1,64 & 1,$16 ; 2,34$ & 1,39 & 0,$87 ; 2,23$ \\
\hline Sul & 1.191 & 46 & 3,9 & 1,20 & 0,$81 ; 1,78$ & 1,07 & 0,$70 ; 1,65$ & 0,85 & 0,$50 ; 1,46$ \\
\hline Centro-0este & 467 & 42 & 9,0 & 2,96 & 1,$96 ; 4,46$ & 3,49 & $2,22-5,46$ & 2,84 & 1,$65 ; 4,90$ \\
\hline \multicolumn{10}{|c|}{ Distância da capital (km) } \\
\hline$\geq 275,0$ & 2.781 & 89 & 3,2 & 1,00 & & 1,00 & & 1,00 & \\
\hline$<275,0$ & 2.789 & 188 & 6,7 & 2,19 & 1,$69 ; 2,83$ & 1,34 & 1,$01 ; 1,78$ & 1,43 & 1,$06 ; 1,92$ \\
\hline \multicolumn{10}{|c|}{ Extensão territorial $\left(\mathbf{k m}^{2}\right)$} \\
\hline$\leq 418$ & 2.785 & 115 & 4,1 & 1,00 & & 1,00 & & & \\
\hline$>418$ & 2.785 & 162 & 5,8 & 1,43 & 1,$12 ; 1,83$ & 0,95 & 0,$72 ; 1,26$ & & \\
\hline \multicolumn{10}{|c|}{ Trabalhadores nas indústrias de transformação/extrativa/construção (\%) } \\
\hline$<15,0$ & 2.974 & 70 & 2,4 & 1,00 & & 1,00 & & & \\
\hline$\geq 15,0$ & 2.596 & 207 & 8,0 & 3,59 & 2,$73 ; 4,74$ & 1,64 & 1,$20 ; 2,25$ & & \\
\hline \multicolumn{10}{|c|}{ Trabalhadores na indústria de transformação (\%) } \\
\hline$\leq 6,4$ & 2.785 & 65 & 2,3 & 1,00 & & 1,00 & & & \\
\hline$>6,4$ & 2.785 & 212 & 7,6 & 3,45 & 2,$60 ; 4,58$ & 1,72 & 1,$26 ; 2,35$ & & \\
\hline \multicolumn{10}{|c|}{ Trabalhadores na indústria extrativa (\%) } \\
\hline$\leq 0,1$ & 2.871 & 114 & 4,0 & 1,00 & & 1,00 & & & \\
\hline$>0,1$ & 2.699 & 163 & 6,0 & 1,55 & 1,$22 ; 1,99$ & 1,18 & 0,$90 ; 1,55$ & & \\
\hline \multicolumn{10}{|c|}{ Trabalhadores na construção (\%) } \\
\hline$\leq 6,0$ & 2.796 & 67 & 2,4 & 1,00 & & 1,00 & & & \\
\hline$>6,0$ & 2.774 & 210 & 7,6 & 3,34 & 2,$52 ; 4,42$ & 1,42 & 1,$03 ; 1,95$ & & \\
\hline \multicolumn{10}{|c|}{ Trabalhadores com emprego formal (\%)e } \\
\hline$\leq 34,1$ & 2.780 & 50 & 1,8 & 1,00 & & 1,00 & & & \\
\hline$>34,1$ & 2.785 & 227 & 8,2 & 4,85 & 3,$55 ; 6,61$ & 2,00 & 1,$41 ; 2,83$ & & \\
\hline
\end{tabular}


Continuação

Tabela 3 - Proporção de municípios ( $\mathrm{N}=5.570)$ que notificaram perda auditiva induzida por ruído (Pair) no Sistema de Informação de Agravos de Notificação, razão de chances e análise multivariada, conforme variáveis contextuais relacionadas ao município, Brasil, 2013-2015

\begin{tabular}{|c|c|c|c|c|c|c|c|c|c|}
\hline \multirow{3}{*}{ Variáveis } & \multirow{3}{*}{ N } & \multirow{2}{*}{\multicolumn{2}{|c|}{ Notificaram Pair }} & \multicolumn{4}{|c|}{ Medida de associação } & \multirow{2}{*}{\multicolumn{2}{|c|}{ Modelo finala }} \\
\hline & & & & \multicolumn{2}{|c|}{ Bruta } & \multicolumn{2}{|c|}{$\begin{array}{c}\text { Ajustada pela } \\
\text { população ocupada }\end{array}$} & & \\
\hline & & $\mathbf{N}$ & $\%$ & $\mathbf{O} \mathbf{R}^{\mathrm{b}}$ & IC $_{95 \%} \mathrm{C}^{\mathrm{C}}$ & $\mathbf{O R}^{\mathrm{b}}$ & IC $_{95 \%}{ }^{\mathrm{C}}$ & $\mathbf{O R}^{\mathbf{b}}$ & IC $_{95 \%}{ }^{\mathrm{C}}$ \\
\hline \multicolumn{10}{|c|}{ Índice de desenvolvimento humano municipal (IDH-M)e,f } \\
\hline Baixo/muito baixo & 1.399 & 16 & 1,1 & 1,00 & & 1,00 & & 1,00 & \\
\hline Médio & 2.263 & 74 & 3,3 & 2,92 & 1,$70 ; 5,04$ & 2,37 & 1,$36 ; 4,11$ & 1,81 & 0,$98 ; 3,32$ \\
\hline Alto/muito alto & 1.903 & 187 & 9,8 & 9,42 & 5,$63 ; 15,77$ & 3,44 & 1,$99 ; 5,94$ & 2,35 & 1,$16 ; 4,75$ \\
\hline
\end{tabular}

a) Ajuste pelo tamanho da população ocupada (Censo 2010) em cinco categorias; b) OR: odds ratio ou razão de chances; c) IC $C_{95 \%}$ : intervalo de confiança de $95 \%$; d) Cerest: (entro de Referência em Saúde do Trabalhador; e) Cinco dados faltantes porque os municípios foram criados após o (enso Demográfico de 2010; f) P-tendência = 0,0175.

Tabela 4 - Proporção de municípios cobertos por Cerest ${ }^{\mathrm{a}}(\mathrm{N}=4.400)$ que notificaram perda auditiva induzida por ruído (Pair) no Sistema de Informação de Agravos de Notificação, razão de chances e análise multivariada, segundo variáveis contextuais relacionadas ao Cerest ${ }^{\mathrm{a}}$ e sua equipe, Brasil, 2013-2015

\begin{tabular}{|c|c|c|c|c|c|c|c|c|c|}
\hline \multirow{3}{*}{ Variáveis } & \multirow{3}{*}{ N } & & & \multicolumn{4}{|c|}{ Medida de associação } & \multirow{2}{*}{\multicolumn{2}{|c|}{ Modelo final ${ }^{b}$}} \\
\hline & & \multicolumn{2}{|c|}{ Notificaram Pair } & \multicolumn{2}{|c|}{ Bruta } & \multicolumn{2}{|c|}{$\begin{array}{c}\text { Ajustada pela } \\
\text { população ocupadab }^{\text {a }}\end{array}$} & & \\
\hline & & N & $\%$ & $\mathrm{OR}^{\mathrm{c}}$ & $\mathrm{IC}_{95 \%}{ }^{\mathrm{d}}$ & $\mathbf{O R}^{\mathrm{c}}$ & IC $_{95 \%}{ }^{d}$ & $\mathrm{OR}^{\mathrm{c}}$ & $\mathrm{IC}_{95 \%}{ }^{\mathrm{d}}$ \\
\hline Municípios cobertos por Cerest ${ }^{a}$ & 4.400 & 254 & 5,8 & & & & & & \\
\hline \multicolumn{10}{|l|}{ Ano de habilitação } \\
\hline$>2005$ & 2.158 & 103 & 4,8 & 1,00 & & 1,00 & & & \\
\hline$\leq 2005$ & 2.242 & 151 & 6,7 & 1,44 & 1,$11 ; 1,86$ & 1,19 & 0,$90 ; 1,59$ & & \\
\hline \multicolumn{10}{|c|}{ Número médio de vínculos (2013-2015) } \\
\hline$<10$ & 1.557 & 70 & 4,5 & 1,00 & & 1,00 & & & \\
\hline$\geq 10$ & 2.843 & 184 & 6,5 & 1,47 & 1,$11 ; 1,95$ & 1,30 & 0,$95 ; 1,77$ & & \\
\hline \multicolumn{10}{|c|}{ Componentes da equipe com vínculo estatutário (\%) } \\
\hline$\leq 50,0$ & 1.926 & 108 & 5,6 & 1,00 & & 1,00 & & & \\
\hline$>50,0$ & 2.474 & 146 & 5,9 & 1,06 & 0,$82 ; 1,36$ & 1,02 & 0,$77 ; 1,36$ & & \\
\hline \multicolumn{10}{|c|}{ Número médio de fonoaudiólogos (2013-2015) } \\
\hline$<1$ & 2.837 & 135 & 4,8 & 1,00 & & 1,00 & & 1,00 & \\
\hline$\geq 1$ & 1.563 & 119 & 7,6 & 1,65 & 1,$28 ; 2,13$ & 1,96 & 1,$47 ; 2,61$ & 1,96 & 1,$47 ; 2,63$ \\
\hline \multicolumn{10}{|l|}{ Rotatividade na equipe } \\
\hline Maior rotatividade (>33,3\%) & 2.261 & 96 & 4,2 & 1,00 & & 1,00 & & 1,00 & \\
\hline Menor rotatividade $(\leq 33,3 \%)$ & 2.020 & 157 & 7,8 & 1,90 & 1,$46 ; 2,47$ & 1,85 & 1,$38 ; 2,47$ & 1,88 & 1,$40 ; 2,52$ \\
\hline
\end{tabular}

a) Cerest: (entro de Referência em Saúde do Trabalhador; b) Ajuste pelo tamanho da população ocupada (Censo 2010) em cinco categorias; c) OR: odds ratio ou razão de chances; d) IC $C_{95 \%}$ : intervalo de confiança de 95\%; e) 119 dados faltantes pela ausência dos registros para 2013 e/ou 2015, ambos necessários para 0 cálculo. 
estruturada da vigilância e do fluxo de notificação dos casos nas áreas de abrangência dos Cerest. Coerentemente, estudo realizado no Brasil sobre a evolução da notificação dos acidentes de trabalho graves no Sinan, entre 2007 e 2011, mostrou a região Centro-0este com a maior proporção de municípios notificantes $(9,7 \%)$ entre as macrorregiões, em 2007, atingindo 31,1\% em 2011, um crescimento expressivo. ${ }^{24}$

No presente estudo, os municípios mais próximos da capital notificaram em maior proporção, comparados àqueles mais distantes. Este achado pode estar relacionado à presença de uma rede de atenção à saúde mais fortalecida, maior acesso dos profissionais a uma educação permanente, presença de técnico de referência em saúde do trabalhador, ações de promoção da saúde e prevenção de doenças e agravos implementadas, maior fiscalização de ambientes de trabalho, e demais fatores contributivos. A proposta de um novo modelo de organização dos Cerest ${ }^{25}$ considera diferentes níveis de referência técnica em saúde do trabalhador, devendo o funcionamento da rede ser planejado de acordo com a faixa populacional dos municípios. Essa reorganização poderá minimizar dificuldades geradas pela distância do município aos grandes centros, com maior capilarização dos serviços, contribuindo para a expansão das notificações em saúde do trabalhador no Sinan. As notificações também podem ser potencializadas nos municípios com a implantação de Comissões Intersetoriais de Saúde do Trabalhador e da Trabalhadora descentralizadas. ${ }^{25}$

Possuir IDH-M elevado mostrou-se também um fator associado à notificação da Pair pelos municípios, com aumento em gradiente. Quanto maior o IDH-M, melhores são as condições de vida, maior 0 acesso ao conhecimento e melhor o padrão de vida medido pela renda municipal per capita. ${ }^{14}$ Estas qualidades podem contribuir para uma boa organização da informação de vigilância em saúde nos territórios. Em contraste, apesar de um baixo IDH, em geral, refletir piores estimativas de morbimortalidade, a notificação de Pair foi menos frequente nos municípios com baixo IDH, o que pode sugerir a hipótese de ser mais comum a subnotificação de casos nos municípios que oferecem uma qualidade de vida ruim a seus cidadãos.

Entre os municípios cobertos por Cerest, ter pelo menos um fonoaudiólogo na equipe foi fator associado à notificação de Pair. Coerentemente, ações de vigilância em saúde direcionadas à Pair e/ou ao distúrbio de voz relacionado ao trabalho foram relatadas pela coordenação dos Cerest como habituais no cotidiano de atuação desses profissionais $(73,7 \%){ }^{26} 0$ fonoaudiólogo, profissional com conhecimento técnico e experiência no manejo de casos de Pair, está habilitado a identificar o tipo de perda auditiva e discutir com a equipe sua possível relação com 0 trabalho. Não obstante 0 crescimento da presença desse profissional nas equipes desde 2002, a maioria das unidades Cerest $(51,9 \%)$ não contava com um fonoaudiólogo em 2014. ${ }^{26}$

Sobre os fatores relacionados aos Cerest de cobertura dos municípios, o mais fortemente associado à notificação da Pair foi a menor rotatividade na equipe de trabalho do Cerest. Ou seja, uma maior rotatividade na equipe parece prejudicar as ações de vigilância. A rotatividade dos profissionais de saúde é um dos problemas a serem enfrentados também nos Cerest, porque interfere na integração da equipe e na atenção aos trabalhadores. ${ }^{27}$ Estudo realizado em Minas Gerais ${ }^{28}$ no período de 2002 a 2007, com foco na atuação dos Cerest regionais, encontrou quase três quartos dos trabalhadores que compunham a equipe dos Cerest com vínculo empregatício efetivo, proporção superior à identificada no presente estudo. A nova proposta de reorganização da Renast indica a necessidade de a equipe multiprofissional, ou a maior parte dela, ser contratada mediante concurso público, com vínculos estáveis. ${ }^{25} \mathrm{~A}$ medida pode contribuir para a menor rotatividade da equipe técnica e gerencial dos Cerest e como resultado, melhor desenvolvimento das ações de vigilância, entre elas as notificações de doenças e agravos no Sinan. Ressalta-se, no entanto, que apesar de a efetividade do vínculo favorecer certa estabilidade na constituição das equipes, ela não garante uma baixa rotatividade de seus componentes, uma vez que pode haver mobilidade na lotação dos trabalhadores.

0 alcance de $100 \%$ das regiões de saúde com no mínimo um Cerest habilitado até 2019 foi uma meta estabelecida em 2015, pelo Plano Plurianual e Plano Nacional de Saúde (2016-2019) do Ministério da Saúde. ${ }^{29}$ Apesar de essa meta não ter sido alcançada, foi publicada a Resolução $n^{0} 603$, de 8 de novembro de $2018,{ }^{25}$ com uma proposta de reorganização da Atenção Integral à Saúde dos Trabalhadores no SUS, cujo objetivo era desenvolver um novo modelo de organização dos Cerest. Nesse modelo, cada Região de Saúde teria, no mínimo, um Cerest regional como retaguarda 
técnica para seus municípios. Considerando-se a relevância do Cerest para a vigilância em saúde do trabalhador, ratificada por este estudo, sua expansão é uma das estratégias para a garantia de apoio institucional, técnico e pedagógico em saúde do trabalhador no território de sua abrangência. Porém, vale ressaltar que existem fatores de impacto no funcionamento dos Cerest, e apenas contar com uma unidade habilitada não garante uma atuação com toda a capacidade potencial, tampouco uma oferta de serviços à população com a equidade, a qualidade e a eficiência que se espera. ${ }^{20}$

Entre as limitações deste estudo, a qualidade dos dados pode ter sido afetada por incompletude e inconsistência, por se tratar de dados secundários de sistemas de informação. Outrossim, a ausência de notificação em municípios muito pequenos, ao contrário de um potencial sub-registro, poderia significar simplesmente a não ocorrência de casos de Pair no período do estudo. Assim, foi empregado o ajuste dos modelos pelo tamanho da população ocupada, como estratégia para minimizar um possível viés nos resultados. Destaca-se que este é o primeiro estudo epidemiológico dedicado a investigar fatores contextuais associados à notificação de Pair no Brasil e adotar, como estratégia, o uso de diversas fontes de dados públicos.

\section{Referências}

1. Nelson DI, Nelson RY, Concha-Barrientos M, Fingerhut M. The global burden of occupational noise-induced hearing loss. Am J Ind Med. 2005 Dec;48(6):44658. doi: https://doi.org/10.1002/ajim.20223.

2. Basner M, Babisch W, Davis A, Brink M, Clark C, Janssen $S$, Stansfeld S. Auditory and non-auditory effects of noise on health. Lancet. 2013 0ct;383(9925):1325-32. doi: https://doi.org/10.1016/S0140-6736(13)61613-X.

3. Sayler SK, Roberts BJ, Manning MA, Sun K, Neitzel RL. Patterns and trends in OSHA occupational noise exposure measurements from 1979 to 2013. Occup Environ Med. 2019 Feb;76(2):118-24. doi: https://doi.org/10.1136/oemed-2018-105041.

4. National Institute for Occupational Safety and Health. Occupational Hearing Loss (ohl) Surveillance: facts and definitions [Internet]. 2018 [citado 26 abr. 2018]. Disponível em: https://www.cdc.gov/niosh/topics/ohl/.

5. Concha-Barrientos M, Nelson DI, Driscoll T, Steenland NK, Punnett L, Fingerhut M, et al. Selected occupational risk factors. In: Ezzati M, Lopez AD,
Fatores contextuais influenciam a notificação de Pair nos municípios. Notadamente, a maioria desses fatores se referem à existência e qualificação dos serviços de atenção à saúde do trabalhador. 0 estudo traz evidências que podem contribuir com o planejamento de estratégias para o fortalecimento da notificação de Pair, e logo, para a redução da subnotificação desse agravo no Sinan. Possíveis recomendações incluem a ampliação do número de Cerest, maior capilarização dos serviços, cursos de capacitação para notificação que alcancem todo o território nacional, maior investimento na vigilância em municípios com baixo IDH, presença de profissional fonoaudiólogo e medidas para reduzir a rotatividade nas equipes dos Cerest.

\section{Contribuição das autoras}

Gusmão AC e Ferrite S contribuíram na concepção e delineamento do estudo, análise e interpretação dos dados e redação da primeira versão do manuscrito. Meira TC contribuiu na análise e interpretação dos dados e revisão crítica do manuscrito. Todas as autoras aprovaram a versão final e são responsáveis por todos os aspectos do trabalho, incluindo a garantia de sua precisão e integridade.

Rodgers A, Murray CJL, organizadors. Comparative quantification of health risks: global and regional burden of disease attributable to selected major risk factors. Geneva: WHO; 2004, p. 1651-801.

6. Santana VS, Silva JM. Os 20 anos da saúde do trabalhador no Sistema Único de Saúde do Brasil: limites, avanços e desafios. In: Ministério da Saúde (BR). Secretaria de Vigilância em Saúde. Departamento de Análise de Situação de Saúde. Saúde Brasil 2008: 20 anos de Sistema Único de Saúde no Brasil. Brasília, DF: MS; 2009. p.175-204.

7. Ministério da Saúde (BR). Portaria ${ }^{0} 2.437$, de 7 de dezembro de 2005. Dispõe sobre a ampliação e 0 fortalecimento da Rede Nacional de Atenção Integral à Saúde do Trabalhador - RENAST no Sistema Único de Saúde - SUS e dá outras providências. Diário Oficial da República Federativa do Brasil. Brasília, DF, 7 dez. 2005 [citado 22 fev. 2018]. Disponível em: http://bvsms.saude.gov.br/bvs/ saudelegis/gm/2005/prt2437_07_12_2005.html 
8. Ferrite S, Meira TC, Santana VS, Cavalcante F, Gusmão AC, Peres MC, et al. Boletim da vigilância dos agravos à saúde relacionados ao trabalho: perda auditiva induzida por ruído ocupacional: informe do centro colaborador PISAT/ISC/UFBA - MS/DSAST/ CGSAT [Internet]. Nov. 2013 [citado 14 mar 2018]. Disponível em: http://renastonline.ensp.fiocruz.br/ sites/default/files/arquivos/recursos/B0L7_PAIR.pdf

9. Guerra MR, Lourenço PMC, Bustamante-Teixeira MT, Alves MJM. Prevalência de perda auditiva induzida por ruído em empresa metalúrgica. Rev Saude Publica. 2005 abr:;39(2):238-44. doi: https:// doi.org/10.1590/S0034-89102005000200015.

10. Caldart AU, Adriano CF, Terruel I, Martins RF, Caldart AU, Mocellin M. Prevalência da perda auditiva induzida pelo ruído em trabalhadores de indústria têxtil. Arq Int Otorrinolaringol. 2006 jul-set;10(3):192-6.

11. Galdino A, Santana VS, Ferrite S. Os centros de referência em saúde do trabalhador e a notificação de acidentes de trabalho no Brasil. Cad Saude Publica. 2012 Jan;28(1):145-59. doi: https:/doi. org/10.1590/S0102-311X2012000100015.

12. Ferreira MJM, Lima, RKS, Silva AMC, Filho JGB, Cavalcanti LPG. Vigilância dos acidentes de trabalho em unidades sentinela em saúde do trabalhador no município de Fortaleza, nordeste do Brasil. Cien Saude Colet. 2017 0ct;22(10):3393-402. doi: https:// doi.org/10.1590/1413-812320172210.17422017.

13. Instituto Brasileiro de Geografia e Estatística (BR) Sistema IBGE de Recuperação Automática. Pesquisa Nacional por Amostra de Domicílios. Tabela 1869: pessoas de 10 anos ou mais de idade, ocupadas na semana de referência, por anos de estudo, sexo e classes de rendimento mensal de todos os trabalhos. [Internet]. 2014 [citado 27 de out. 2020]. Disponível em: https://sidra.ibge.gov.br/Tabela/1869

14. Programa das Nações Unidas para o Desenvolvimento. Atlas de desenvolvimento hmano no Brasil [Internet]. 2013 [citado 21 jun. 2019]. Disponível em: http:// www.atlas brasil.org.br/2013/pt/o_atlas/idhm/

15. Instituto Brasileiro de Geografia e Estatística (BR). Conheça cidades e estados do Brasil [Internet]. 2017. Salvador: IBGE; 2018 [citado 14 nov. 2018]. Disponível em: https://cidades.ibge.gov.br/

16. Google. Google Maps [Internet]. 2018 [citado 14 nov. 2018]. Disponível em: https:// www.google.com.br/maps
17. Centro Colaborador da Vigilância aos Agravos à Saúde do Trabalhador. Sistema de Informação de Agravos de Notificação - Sinan. Banco de notificações de pair no Sinan [Internet]. 2018 [citado 14 nov. 2018]. Disponível em: http://www.ccvisat.ufba.br/

18. Rede Nacional de Atenção à Saúde do Trabalhador. Centros de Referência em Saúde do Trabalhador [Internet]. 2018. Salvador: Centros de Referência em Saúde do Trabalhador; 2014 [citado 14 nov. 2018]. Disponível em: http://renastonline.ensp.fiocr uz.br

19. Cadastro Nacional de Estabelecimentos de Saúde (BR). Consulta estabelecimento - identificação [Internet]. 2018. Salvador: Cadastro Nacional de Estabelecimentos de Saúde; 2013-2015 [citado 14 nov. 2018]. Disponível em: http://cnes.datasus. gov.br/pages/estabelecimentos/consulta.jsp

20. Ministério da Saúde (BR), Fundação Oswaldo Cruz, Universidade Federal da Bahia. $2^{\circ}$ Inventário de saúde do trabalhador, 2010-2011: acompanhamento da Rede Nacional de Atenção Integral em Saúde do Trabalhador, 2010-2011. [Internet]. Brasília, DF. 2013 set [citado 4 maio 2019]. 138 p. Disponível em: http://renastonline. ensp.fiocruz.br/sites/default/files/arquivos/recursos/ Inventario\%20RENAST\%202010-2011.pdf

21. Ministério da Saúde (BR). Portaria n ${ }^{\circ}$ 2.978, de 15 de dezembro de 2011. Amplia para 210 (duzentos e dez) a quantidade de Centros de Referência em Saúde do Trabalhador (CEREST) passíveis de implantação no território nacional. Diário Oficial da República Federativa do Brasil. Brasília,DF; 15 dez. 2011. [citado 4 maio 2019]. Disponível em: http://bvsms.saude.gov.br/ bvs/saudelegis/gm/2011/prt2978_15_12_201 1.html

22. Bezerra MLS, Neves EB. Perfil da produção científica em saúde do trabalhador. Saude Soc. 2010 Jun;19(2):384-394. doi: http://doi. org/10.1590/S0104-12902010000200014.

23. Secretaria de Estado da Saúde de São Paulo. Centro de Referência em Saúde do Trabalhador de São Paulo. Processo de implantação da saúde do trabalhador no SUS/SP. Rev Saude Publica. 2004 jun;38(3):471-4. doi: http://dx.doi.org/10.1590/S0034-89102004000300021.

24. Bastos-Ramos TP, Santana VS, Ferrite S. Estratégia saúde da família e notificações de acidentes de trabalho, Brasil, 2007-2011. Epidemiol Serv Saude. 2015 out.-dez.;24(4):641-50. doi: http://dx.doi. org/10.5123/S1679-49742015000400006. 
25. Ministério da Saúde (BR). Resolução n ${ }^{0}$ 603, de 8 de novembro de 2018. Rede Nacional de Atenção Integral à Saúde do Trabalhador - Proposta de Reorganização das Ações e Serviços de Saúde do Trabalhador na Rede SUS. Brasília, DF: MS; 2018 nov. 8. [citado 4 maio 2019]. 40 p. Disponível em: https://renastonline.ensp.fiocruz.br/sites/ default/ files/arquivos/recursos/resoluca_n_603_de_8_ de_novembro_de_2018_cns_cerest_renast.pdf

26. Gusmão AC, Meira TC, Santos FCCN, Ferrite S. A Fonoaudiologia nos centros de referência em saúde do trabalhador no Brasil. Rev CEFAC. 2018 nov.-dez:;20(6):723-33. doi: http://doi; org/10.1590/1982-0216201820621117.

27. Santos APL, Lacaz FAC. Apoio matricial em saúde do trabalhador: tecendo redes na atenção

\section{Abstract}

Objective: To investigate factors associated with the notification of noise-induced hearing loss (NIHL) on the Notifiable Health Conditions Information System (SINAN) in Brazil. Methods: This was an ecological study to estimate the proportion of municipalities that had notified NIHL. Logistic regression models were used to identify associated factors. Results: Between 2013-2015, 277 (5.0\%) municipalities notified NIHL. Notification was more prevalent among municipalities in an Occupational Health Referral Center (CEREST) coverage area $(O R=1.62$ - 95\%CI 1.02;2.59) or those that had a CEREST in their territory (OR=4.37 95CI\% 2.75;6.93), those that were closer to the state capital (OR=1.43 - 95\%CI 1.06;1.92) and those with a bigh buman development index (OR=2.35 - 95\%CI 1.16;4.75). Among the municipalities located in a CEREST coverage area, notification was more frequent when there was a speech-language-hearing professional in the team (OR=1.96 - 95\%CI - 1.47;2.63) and when employee turnover was low $(O R=1.88-95 \% C I$ 1.40;2.52). Conclusion: Contextual factors influence NIHL notification, particularly the presence of CERESTs and their qualification.

Keywords: Hearing Loss, Noise-Induced; Health Information Systems; Public Health Surveillance; Occupational Health; Ecological Studies. básica do SUS, o caso de Amparo/SP. Cienc Saude Colet. 2012;17(5):1143-50. doi: http:// doi.org/10.1590/S1413-81232012000500008.

28. Dias EC, Chiavegatto CV, Silva TL, Reis JC, Silva JM. Construção da RENAST em Minas Gerais: a contribuição dos centros de referência em saúde do trabalhador (CEREST), 2002-2007. Rev Med Minas Gerais. 2010;20( Suppl 2):66-74.

29. Ministério da Saúde (BR). Planejamento estratégico da RENAST: reunião com os coordenadores estaduais de saúde do trabalhador [Internet]. [Brasília, DF]: 2015 [citado 4 maio 2019]. 4 p. Disponível em: http://renastonline.ensp.fiocruz.br/sites/ default/files/arquivos/p aginas/Planejamento\%20 Estrategic0\%20da\%20Renast_Reuni\%C3\%A30\%20 dos\%20C oordenadores\%20da\%20Renast.pdf

\section{Resumen}

objetivo: Investigar factores asociados con la notificación de pérdida auditiva inducida por ruido (Pair) en el Sistema de Información de Agravamientos de Notificación Obligatoria en Brasil. Métodos: Estudio ecológico para estimar la proporción de municipios notificantes de Pair. Se utilizaron modelos de regresión logística para identificar factores asociados. Resultados: Entre 2013-2015, 277 (5,0\%) municipios notificaron Pair. La notificación fue más común entre los municipios con cobertura de los Centros de Referencia en Salud Ocupacional (Cerest) $\left(O R=1,62-I C_{95 \%} 1,02 ; 2,59\right)$, o que albergaron Cerest $\left(O R=4,37-I C_{95 \%} 2,75 ; 6,93\right)$, cercanos a la capital $\left(O R=1,43-I C_{95 \%} 1,06 ; 1,92\right) y$ con alto indice de desarrollo bumano (OR=2,35 - IC ${ }_{95 \%}$ 1,16; 4,75). Entre los municipios en área de cobertura de Cerest, la notificación fue más alta cuando babía fonoaudiólogo en el equipo (OR=1,96-IC $\left.{ }_{95 \%} 1,47 ; 2,63\right)$ $y$ baja rotación de profesionales $\left(O R=1,88-I C_{95 \%} 1,40\right.$; 2,52). Conclusión: Factores contextuales influyen en la notificación de Pair, en particular, la existencia y cualificación de los Cerest.

Palabras clave: Pérdida Auditiva Provocada por Ruido; Sistemas de Información en Salud; Vigilancia en Salud Pública; Salud Laboral; Estudios Ecológicos.

Recebido em 09/08/2020

Aprovado em 21/12/2020

Editor associado: Bruno Pereira Nunes - (1) orcid.org/0000-0002-4496-4122 Editora científica: Taís Freire Galvão - (1) orcid.org/0000-0003-2072-4834 Editora geral: Leila Posenato Garcia - (1) orcid.org/0000-0003-1146-2641 